\title{
The Case for Bi-directional Charging of Electric Vehicles in Low Voltage Distribution Networks
}

\author{
Constance Crozier ${ }^{\mathrm{a}, *}$, Thomas Morstyn ${ }^{\mathrm{a}}$, Matthew Deakin ${ }^{\mathrm{a}}$, \\ Malcolm McCulloch ${ }^{\mathrm{a}}$ \\ ${ }^{a}$ Department of Engineering Science, University of Oxford, Parks Road, OX1 3PJ
}

\begin{abstract}
In recent years, there has been growing interest in $\mathrm{AC}$ vehicle-to-grid charging in residential networks. Bi-directional smart charging offers additional flexibility compared to uni-directional smart chargers, but are expensive to install and implement. This paper investigates the costs and benefits to the distribution network that bi-directional charging provides, relative to unidirectional charging. Benefit is quantified in terms of the reduction in the peak demand of the network, and costs are quantified with the increase in throughput of the vehicles' batteries and the increase in electrical losses in the system. Measured data and representative networks are used to construct two representative case studies of residential charging, one in the UK and one in Texas, US.
\end{abstract}

Keywords: Electric vehicles, Losses, Low-voltage Networks, Vehicle-to-grid

\section{Introduction}

This paper investigates the costs and benefits to the system of smart bidirectional charging of electric vehicles, compared to smart uni-directional charging, in low voltage residential networks.

Electric vehicles $(\mathrm{EVs})$ are becoming more prevalent in developed countries, and charging at home appears to be the preferred option [1]. Home chargers are connected to low-voltage networks, which typically supply between 20 and 200 households with electrical power [2]. EV charging is expected to create problems for these networks by overloading transformers [3]

\footnotetext{
${ }^{*}$ Corresponding author.
} 
and creating unacceptably low voltages [4]. As a result, the UK government has announced that all home charge points must be smart from July 2019 [5]. Smart charging refers to manipulating the time and size of the charging load in order to improve the system operation, typically by reducing the peak demand on the network (e.g. [6, 7]). Uni-directional smart chargers do this by simply shifting the load to an off-peak time, but peak load can be further reduced using bi-directional smart charging [8]. In this case vehicles are able to send power back to the grid at peak times, which is often referred to providing as vehicle-to-grid (V2G) services.

As of Q1 2019, the majority of commercially available V2G chargers are DC fast chargers - designed to be connected directly to the higher voltage network. However, trials have been taking place using AC low power V2G chargers [9], which could be connected into residential networks. The technology required for bi-directional charging is more complex and expensive than that for uni-directional charging. Additionally, in order for users to provide power to the network, a market framework must be set up. Therefore, careful consideration needs to be given to the value that V2G adds to the local system before this technology is pursued.

Considering the value of $\mathrm{V} 2 \mathrm{G}$ requires defining the system which the vehicle is operating within. Some studies focus only on the value gained by the owner of the individual vehicle [10], a fleet owner [11], or the network operator [12]. However, in order for V2G to be worthwhile, it must add value to the system as a whole - e.g. if all benefit to the individual is counter-balanced by a cost to the network operator then the technology is not viable.

The number of vehicles present in the system may also have an effect on the results. The demand flexibility increases with the number of vehicles, but so does the total energy demand on the network. Therefore there is likely to be a trade-off, meaning there is some penetration level where the benefit of V2G is optimised. Previous work has only considered a small number of discrete penetration levels (e.g. 15, 35, and $75 \%$ in [8]). Therefore, here consumers with and without vehicles, at various ratios, are considered in the analysis.

The hardware expense is not the only additional cost incurred from V2G. Round-trip losses in the charger mean that the households' total net energy consumption will increase [13] - which, depending on the buy and sell price, may make the service unprofitable. Several studies have attempted to quantify these costs by making assumptions about the future market framework, 
however, the results varied significantly depending on the market mechanism assumed; [10] finds that V2G is viable when there is a large difference in sell and buy price, while others found that participation in the frequency [14] or balancing [11] markets are required to produce a profit. The electricity prices also have a large effect on the results; [15] suggests that based on the UK market buy and sell prices there is a case for V2G, [16] finds that there is small value to be gained using the German market but none in the Swedish market, and [17] concludes that in the Spanish market the differences between costs and income are so small that further investigation is required.

There is also doubt over whether V2G provision is the most cost effective method to reduce overloads. In [18] is it concluded that if the components are severely overloaded then it is more cost effective to replace them. Furthermore, on the basis of an analysis of the Danish power system, [19] concludes that EVs could negatively impact system stability if too many were allowed to provide ancillary services.

Additionally, the total throughput of EVs' batteries will be increased, which may accelerate their ageing. An EV's battery accounts for 15-35\% of its total cost, and batteries must be replaced once their capacity has reached around $80 \%$ of its initial capacity. There is a lack of consensus around the impact of V2G on battery degradation; [20] concludes that battery degradation will significantly reduce the revenue for $\mathrm{V} 2 \mathrm{G}$ and [21] found that it made V2G unviable, whereas [22] and [23] suggest that V2G may increase the lifetime of batteries if properly managed. This presents a problem when analysing the financial case of $\mathrm{V} 2 \mathrm{G}$, so in this paper the costs are considered in terms of the increase in battery throughput, which is widely used as a first order model for cycle ageing [24].

The aim of $\mathrm{V} 2 \mathrm{G}$ is to lower the network costs, by reducing the peak power demand. However, if EVs are supplying power to other households, then the currents throughout the network are likely to increase - resulting in more resistive losses. As these losses are in front of the households' electricity meters, the costs are incurred by the network operators - who will pass it onto customers using the tariff rate. It is therefore important to consider these losses separately to those occurring in chargers, as they are paid for by all customers - regardless of whether they have an EV or not.

Consideration also needs to be given to how the benefits of V2G are defined. The majority of the studies previously mentioned consider the potential increase in profit made by the owner of the EV. However, this depends on the pricing and market framework, which have not yet been decided. There- 
fore, it is the value that V2G adds to the system which should be quantified. Once this has been determined, a market framework can be set up to incentivise EVs to participate. Suggested uses of V2G include the islanding of micro-grids [25, 26] and increased use of renewables [27]. However, here the benefit is quantified in terms of the additional reduction in peak demand on the network, when compared to uni-directional smart charging. The peak demand on the network determines the required transformer rating, and large peak demands result in large losses and voltage drops. Therefore, peak demand reduction is a key aim of demand response programs (e.g. [28]).

Although various costs and benefits of V2G have been quantified under specific conditions, a comprehensive study taking into account all of the costs and benefits mentioned has not been carried out. This paper fills this gap in the literature by providing a rigorous analysis of the costs and benefits of bidirectional smart charging, when compared to uni-directional smart charging. This is achieved through two stochastic case studies, using representative networks with measured household and vehicle charging data.

The contributions of this paper can be summarised as follows. First, that a rigorous analysis of the costs and benefits of including bi-directional charging in residential distribution networks is presented. Second, that costs are separated into those incurred by the EV owner, and those incurred by the system. Third, that the results are presented as a function of EV penetration level, investigating the evolution of the value of $\mathrm{V} 2 \mathrm{G}$ as population of EVs increases.

The remainder of this paper is structured as follows. Section 2 details the methodology used in this analysis; data sources in Sections 2.1 and 2.2, test networks in 2.3, the uni-directional and bi-directional optimisation problems in Sections 2.4.1 and 2.4.2, the model for losses in Section 2.5, and the modelling framework in Section 2.6. The results of the two case studies are presented in Section 3, and Section 4 concludes the paper.

\section{Modelling Methods}

In this section, the data sources and modelling methods used in this study are described. First, the household and vehicle demand data sources are described. Then, the optimisation problems are formulated, and the method of calculating losses is explained. Finally, the modelling framework used to create stochastic results is described. 


\subsection{Household Demand}

For the UK data, 30 minute resolution smart meter data was used from 14,000 households in the UK recorded between 2007 and 2010 [29]. These households contain a mix of single and dual tariff customers from all over the country, most of whom are also connected to the gas network. In 2010 there were less than 100 plug-in EVs registered in the UK, so it is assumed that EV charging was not included in the demand data. Figure 1a shows the average Wednesday demand profile in the data set, along with bounds covering $80 \%$ of the data. The average peak at this time resolution is 0.4 $\mathrm{kW}$ per household and the confidence interval covers only positive values, implying there was not a significant amount of generation present.

For the Texas case, data from the Pecan Street project was used, which includes appliance level and total household demand data [30]. Only data from Texan households, recorded on Wednesdays from summer 2018 was used. Some households owned EVs, however their charging was metered separately so could be removed from the profiles. Several of the households had solar panels, and their generation was included in the demand profiles. Figure $1 \mathrm{~b}$ shows the average profile from the dataset. The average peak is around $3 \mathrm{~kW}$ per household, more than 6 times the peak from the UK data - this difference can be attributed largely to the additional load from air conditioning. The confidence interval also covers negatives values up to -2 $\mathrm{kW}$, meaning the households sometimes become net exporters.

\subsection{Vehicle Usage}

Due to the novelty of the technology, EV usage data is currently scarce. While some small scale trials exist, these are likely to represent a biased set of customers; [31] reports that EV early-adopters are typically male, highly educated, have above average incomes, and often own more than one vehicle. Therefore, large-scale and population-representative travel survey data recording the behaviour of conventional vehicles is used. This allows a much more diverse range of driving behaviour to be captures, but necessitates the assumption that electrification will not significantly change driving behaviour.

For the UK data, the National Travel Survey was used [32]. This recorded the trip times and distances of 160,000 vehicles over a week long survey period. The Texas data was extracted from the US National Household Travel Survey which recorded similar parameters, but only over a single day 


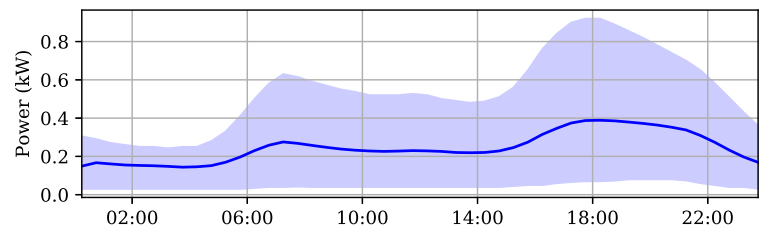

(a) UK Smart Meter Data

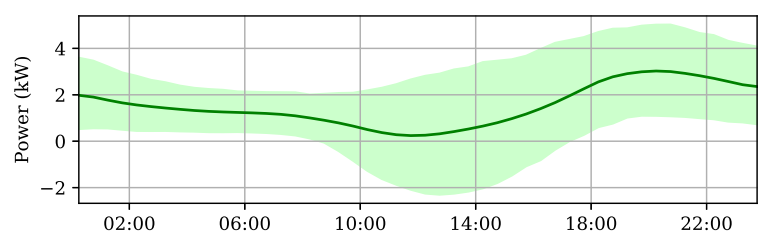

(b) Texas Pecan Street Data

Figure 1: The average household profile in each dataset. The solid lines show the mean value and the shaded area covers the $80 \%$ confidence interval.

survey period [33]. The dataset included just under 60,000 vehicles from Texas.

In order to predict the energy consumption of the vehicle, distance travelled must be converted into an energy expenditure, and for this study a conversion of $0.3 \mathrm{kWh} /$ mile was used - which is equivalent to the tested fuel economy of a Nissan Leaf [34]. Figure 2a compares the probability distribution of daily distance between the two datasets. The US vehicles travel further on average, but in both cases a considerable portion of the fleet is unused on any given day.

Journey purposes are also recorded, so it is possible to infer the range of times that the vehicle is at home. As these are simulations of residential charging, it is assumed that the vehicle can only charge during these times. Figure $2 \mathrm{~b}$ shows the probability that a vehicle from either dataset is home throughout the day. The probability distributions are similar, although the UK vehicles are slightly more likely to be available in the middle of the day.

\subsection{Test Networks}

Assessing the impact of EV charging on distribution system losses requires a representative test network. Both the base voltage and the network style vary between the UK and the US so separate networks were used for each case study. For the UK case study, the IEEE European Low Voltage Test 


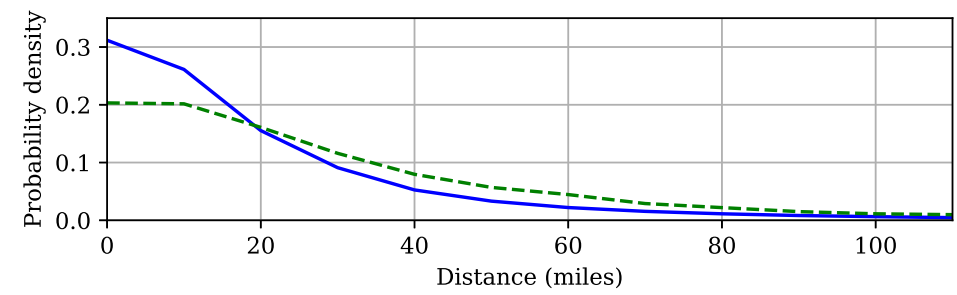

(a) Distribution of daily driven distance.

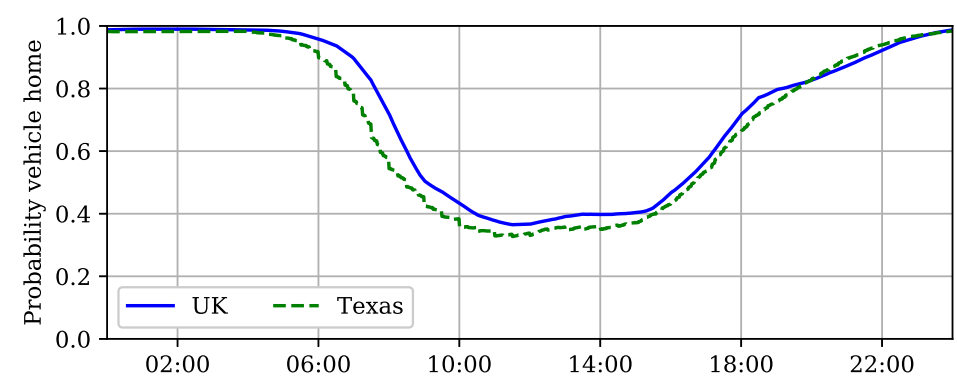

(b) Probability of a vehicle being at home with time of day.

Figure 2: A comparison of the driving behaviour exhibited in the two travel surveys.

Feeder is used (see Figure 3a). There are 55 loads on the system, each of which represent a single residential household. For the Texas case study, Feeder K1 from the EPRI set of test networks is used [35]. This network (shown in Figure 3b) has 311 residential loads, and 13 industrial loads. It was assumed that the industrial loads were fixed and constant with time.

\subsection{Optimisation Problem}

In order to compare the best-case action of both V2G and G2V schemes, optimisation problems are required to compute the optimal solution is each case. The objective is to minimise the peak demand once all vehicle charging has been incorporated. If only G2V charging is considered, then this can be achieved by minimising the 2-norm of the load. However, in the V2G case the charger losses mean that the total energy required by the feeder depends on the charging profiles - so flattening the load and minimising the 2-norm are not equivalent. In order to arrive at a convex formulations, it is assumed that charging efficiency is given by a constant, $\eta_{c}$ for charging and $\eta_{d}$ for discharging. 


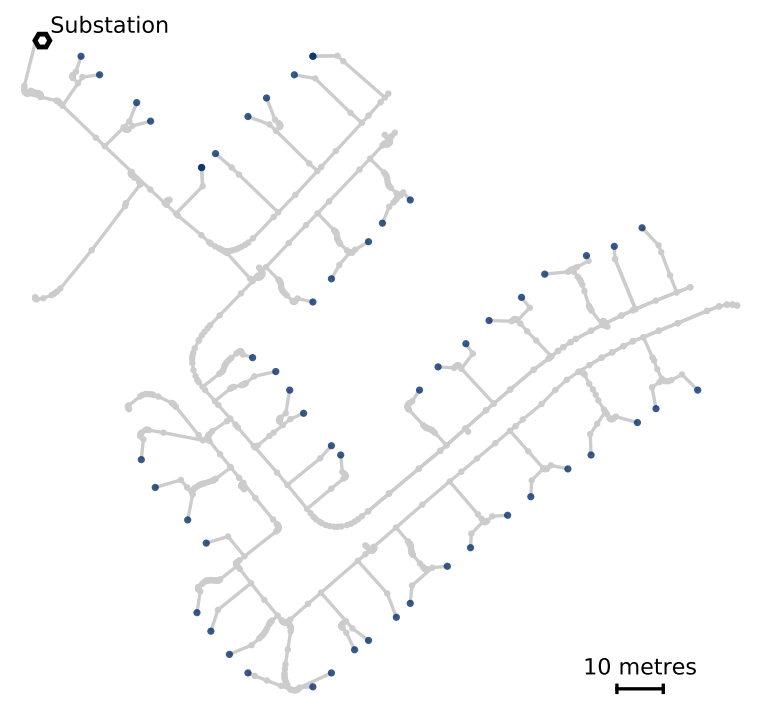

(a) The IEEE European Low Voltage Test Feeder

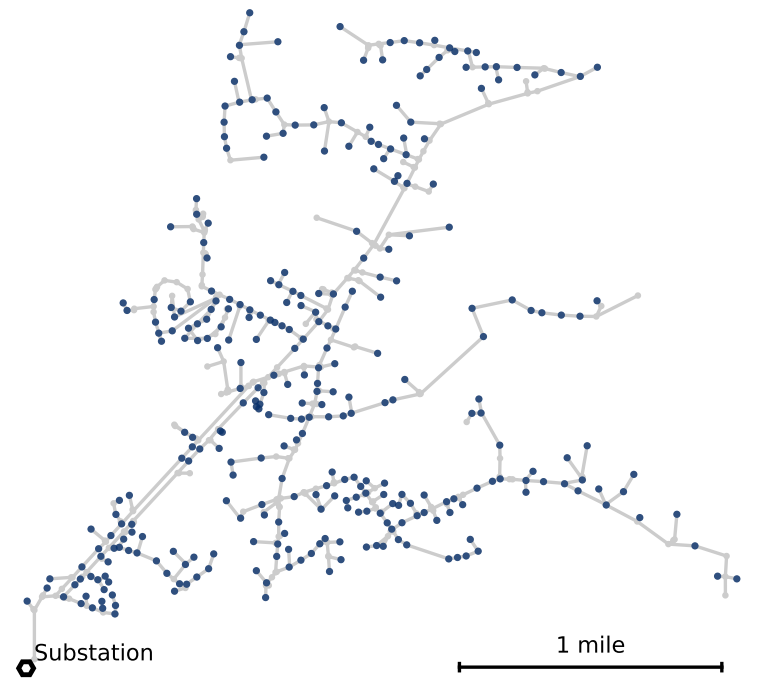

(b) EPRI Network

Figure 3: The test networks considered, the blue markers show the positions of the loads.

\subsubsection{Uni-directional Charging}

Consider a fleet of $N$ vehicles over $T$ discrete time intervals of duration $\Delta t$. The charging power of the vehicle at node $j$ during time interval $t$ is given 
by $x_{t}^{(j)}$. The vector $\mathbf{x}^{(j)} \in \mathbb{R}^{T}$ then represents the proposed charging profile of that vehicle over the whole time period, and $\mathbf{h}^{(j)}$ is the household's existing demand profile. In order to minimise peak load, an additional variable $z$ is defined such that:

$$
p_{t}+\sum_{j=1}^{N} x_{t}^{(j)} \leq z \forall t,
$$

where $p_{t}$ is the total load on the feeder at time $t$. The objective of the optimisation is then described by:

$$
\min _{\mathbf{x}, z} z
$$

In addition to (1) the optimisation is subject to the following constraints:

$$
\begin{gathered}
\sum_{t=1}^{T} \eta_{c} x_{t}^{(j)} \Delta t=E^{(j)}, \\
0 \leq x_{t}^{(j)} \leq P_{\max } \forall j, t, \\
x_{t}^{(j)}=0 \text { for } t \in \mathbb{T}^{j}
\end{gathered}
$$

where $E^{(j)}$ is the energy required by vehicle $j, P_{\max }$ is the maximum possible charging power (which is set by the charger, and therefore the same for all vehicles), and $\mathbb{T}^{j}$ is the set of times for which vehicle $j$ is unavailable to charge. This means that (3) ensures that each vehicle has received the right amount of energy at the end of the time horizon, (4) limits each charging power to be non-negative and below a maximum value, and (5) prevents a vehicle from charging when it is unavailable.

\subsubsection{Bi-directional Charging}

In order to allow bi-directional charging, the problem described in Section 2.4.1 must be altered. It is insufficient to merely remove the positive constraint on vehicle charging, as the charger's round-trip efficiency must be taken into account. 
Here two sets of variables are defined, $x_{t}^{(j)}$ for the power flow to EV $j$ at time $t$, and $y_{t}^{(j)}$ for the power from $\mathrm{EV} j$ at time $t$. This means the constraint (1) can be rewritten as:

$$
p_{t}+\sum_{j=1}^{N} x_{t}^{(j)}-\sum_{j=1}^{N} y_{t}^{(j)} \leq z \quad \forall t
$$

and (3) as:

$$
\sum_{t=1}^{T}\left(\eta_{c} x_{t}^{(j)}-\frac{y_{t}^{(j)}}{\eta_{d}}\right) \Delta t=E^{(j)} .
$$

An additional constraint is also required to ensure that the SOC of the vehicles remains between 0 and $100 \%$ for all time instances. Mathematically this can be written as:

$$
0 \leq E^{(j)}+\sum_{t=1}^{\tau}\left(\eta_{c} x_{t}^{(j)}-\frac{y_{t}^{(j)}}{\eta_{d}}\right) \leq C^{(j)} \quad \forall \tau,
$$

where $C^{(j)}$ is the capacity of vehicle $j$ in $\mathrm{kWh}$. The constraints (4-5) can be extended for all $y$ as well as $x$, and the objective becomes:

$$
\min _{\mathbf{x}, \mathbf{y}, z} z
$$

This formulation will implicitly prioritise charging over discharging for flattening loads, due to the efficiency terms. In other words, $y_{t}^{(j)}$ will be necessarily zero if $x_{t}^{(j)}$ is non-zero. This is because any non-zero $y$ will increase the total energy demand, so it will only be increased when it directly reduces the objective - i.e. at peak demand time. This means that there will no times when there is both charging and discharging.

\subsection{Losses Model}

It is necessary to model the losses which will occur in both the charger and throughout the network. This section describes the models used to quantify losses in this paper.

Charging losses account for the difference between the DC power into the EV battery and the AC power drawn from the grid. The owner of the charge point pays for the metered $\mathrm{AC}$ power drawn from the grid, so these losses 
are paid for by the householder. These losses occur in the converter and can be broken down into conductive losses (which vary quadratically with load), and switching losses (which are approximately constant) [36]. Therefore, charging slowly results in a low efficiency because the switching losses make up a proportionally larger amount of the load. In [37] a bi-directional EV charger was operated at various power levels and the efficiency was recorded in each case - the efficiency in both directions was approximately constant above $50 \%$ of the rated power, but dropped rapidly below this. Here a constant charging efficiency is required to maintain the convex optimisation formulation. It is noted that if the optimal profiles are low power then this may result in vehicles not achieving the required state-of-charge.

With the constant efficiency assumption, the total charging losses of vehicle $j$ can be calculated as:

$$
\left(1-\eta_{c}\right) \sum_{t}^{T} x_{t}^{(j)}+\left(1-\eta_{d}\right) \sum_{t}^{T} y_{t}^{(j)}
$$

Resistive losses are proportional to the square of current, therefore high peak loads tend to result in large resistive losses. These losses occur in front of the meter, meaning they are not billed to an individual consumer. Instead, these losses will be paid for as part of the fee taken by network operators - meaning that all customers on the network pay for any additional losses, regardless of whether or not they have an EV. The (complex) nodal current injections and voltages are found using the power flow method described in [38], and the resistive losses are then calculated as:

$$
\sum_{t}^{T} \sum_{k}^{K} \operatorname{Re}\left\{v_{t}^{(k)} i_{t}^{*(k)}\right\}
$$

where $v_{t}^{(k)}$ is the voltage at bus $k$ during time $t$, and $i_{t}^{*(k)}$ is the complex conjugate of the current at bus $k$ during time $t$.

\subsection{Modelling framework}

Monte Carlo simulations are used to incorporate stochasticity. This involves running a large number of simulations, where each of the stochastic inputs are randomly chosen each time. In this case, the input variables are the household loads, and vehicle usage on the network. The number of EVs 
on the network is assumed to be known (deterministic). Therefore, probability distributions can be estimated for peak demand, battery throughput, and losses. The simulations were run in Python, interfacing with the power flow solver OpenDSS[39].

In each simulation instance, vehicles and loads from the data sets are randomly added to the network. Household and vehicle data from Wednesdays at the same time of year are selected, to incorporate seasonal and weekly dependance of loads. Then both V2G and G2V optimisation problems are run, resulting in two sets of optimal charging profiles. These are used to calculate the distribution and charging losses in each case, and the peak demand, average battery throughput, and losses are all recorded. After a large number of simulations have been carried out, the results are used to form a discrete probability distribution.

Many of the results presented in this paper consider the percentage difference between the uni-directional and bi-directional results. For these the uni-directional charging is considered the base case, so the percentage change in quantity $x$ is given by:

$$
\frac{x_{b}-x_{u}}{x_{u}} \times 100
$$

where $x_{b}$ is the quantity in the bi-directional case and $x_{u}$ is the quantity in the uni-directional case. For losses it was decided to use the absolute difference rather than the percentage change, because a financial cost can be easily assigned to electricity losses - whereas it is very difficult to quantify the cost of a reduction in peak demand or an increase in battery throughput.

This procedure is repeated for a variety of EV penetration levels. Here a $100 \%$ penetration is defined to mean that there is one EV per household on the network.

\section{Results \& Discussion}

In this section the results from the UK and Texas case studies are presented. In each case, the possible reduction in peak demand is quantified for a variety of levels of EV penetration, along with the associated increases in battery throughput and system losses. The simulations were run as described in Section 2.6, with a period of one day split into 30 minute intervals - meaning that $T=48$. Values of $90 \%$ were used for both charging and discharging efficiency, as similar values have been observed in home EV charging [40]. 

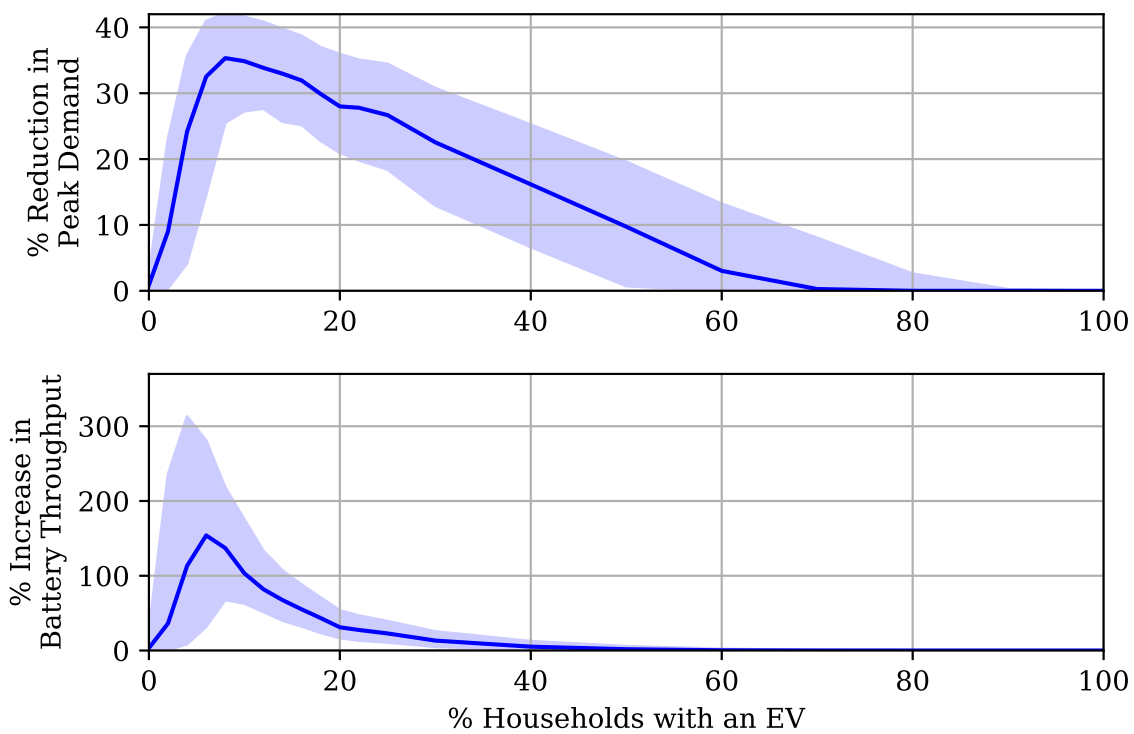

Figure 4: The additional reduction in peak demand achieved by bi-directional over unidirectional smart charging in the UK, varying with EV penetration. Percentages are calculated according to (12). The solid lines show the average and the shaded area covers the $90 \%$ confidence interval.

\subsection{UK Case Study}

The UK case study used a European style 55 household distribution system. Figure 4 shows the percentage reduction in peak demand achieved by bi-directional over uni-directional smart charging, and the associated increase in battery throughput of the EVs, against the percentage of households on the network which had an EV. The solid line shows the average values over the Monte Carlo simulations, and the shaded area covers the $90 \%$ confidence interval. A confidence interval is a measure of the observed variance in the data, as it covers $90 \%$ of the observed values for that EV penetration.

With a very small number of electric vehicles, the average reduction is low, and the variance is high. This is because there is a significant chance that there will be no vehicles available to discharge at peak times. The additional reduction in peak demand is largest when approximately $10 \%$ of the households have EVs, with an average of $35 \%$ reduction possible. Once the EV population grows beyond this point the additional reduction decays away, leaving no additional reduction possible when $100 \%$ of households have 


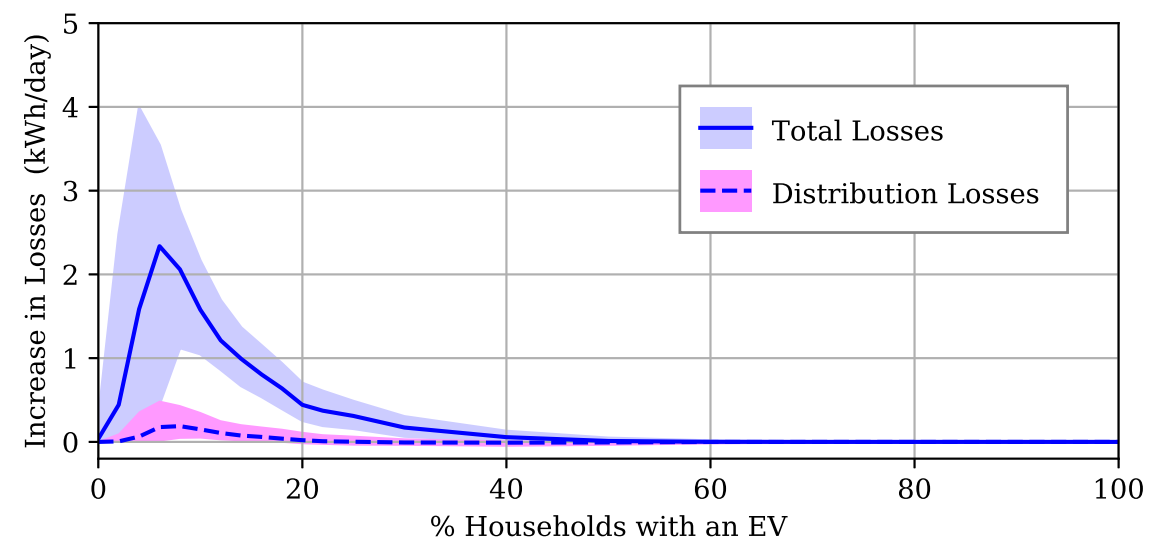

Figure 5: The increase in total losses and those just in the distribution system using bidirectional charging compared to uni-directional. The lines show the average values and the shaded areas cover the $90 \%$ confidence interval.

an EV. This is because additional vehicles add load, as well as flexibility, to the network - at $100 \%$ the load can be perfectly flattened using unidirectional charging. The increase in battery throughput is closely related to the reduction in peak demand, which is intuitive as both are dictated by the amount of V2G deployed. At the point of maximum V2G, vehicles' throughout is increased by an average of $150 \%$. The variance in battery throughput is larger than the variance in peak demand reduction, as battery throughput is dependant on the distance travelled by the vehicles as well as the amount of $\mathrm{V} 2 \mathrm{G}$ provided.

Figure 5 shows the change in losses for the same set of simulations. The solid line shows the total losses, and the dotted line shows the distribution losses - the charging losses account for the difference between the two. The lines show the average values, and the shaded area covers the $90 \%$ confidence interval. Both charging and resistive losses were always increased by the addition of $\mathrm{V} 2 \mathrm{G}$, despite the reduction in peak demand. This is because the peak load dictates the current at the top of the feeder, but not elsewhere in the network. In this case the charging losses are much more significant than the distribution losses. This adds to the costs paid by the EV owner, on top of the increase in battery degradation. 

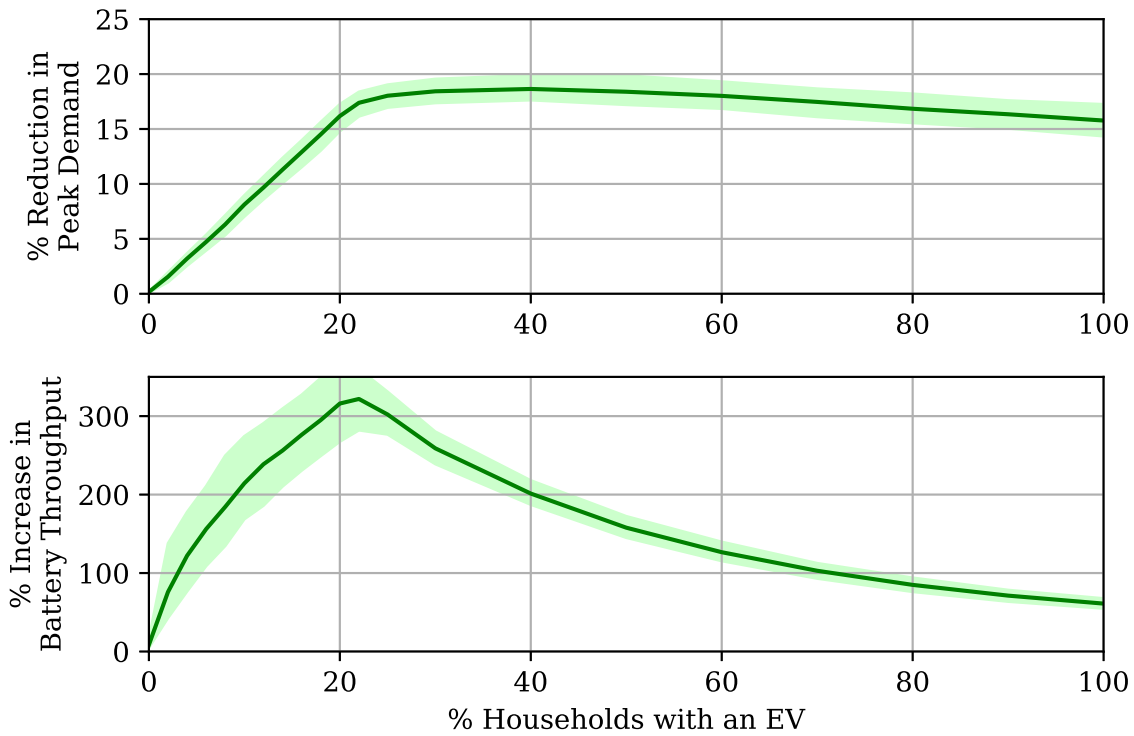

Figure 6: The additional reduction in peak demand achieved by bi-directional over unidirectional smart charging in Texas, varying with EV penetration. Percentages are calculated according to (12). The solid lines show the average and the shaded area covers the $90 \%$ confidence interval.

\subsection{Texas Case Study}

The second case study focused on a US style network using vehicles and household loads from Texas, some of which included solar generation. The percentage reduction in peak demand and increase in battery throughput are shown in Figure 6. In this case a maximum reduction in peak demand of 15-20\% occurred when $25 \%$ of households had EVs. The variation between simulation runs was lower than in the previous case, which is unsurprising given there are a larger number of loads on the network.

Similar to the UK case, when there are a very small number of vehicles, availability limits the potential reduction. However, unlike the UK case, there is still additional value to bi-directional charging when $100 \%$ of households have vehicles. This is because the solar generation and higher household loads mean there is a much larger difference between peak and trough demand, so the EV load is not large enough to flatten the load with only uni-directional charging. This is demonstrated in Figure 7, which shows examples of the total feeder load profiles when $100 \%$ of households had an EV. The dotted 


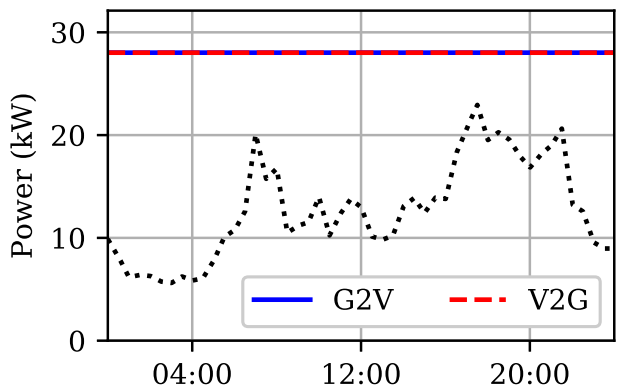

(a) UK

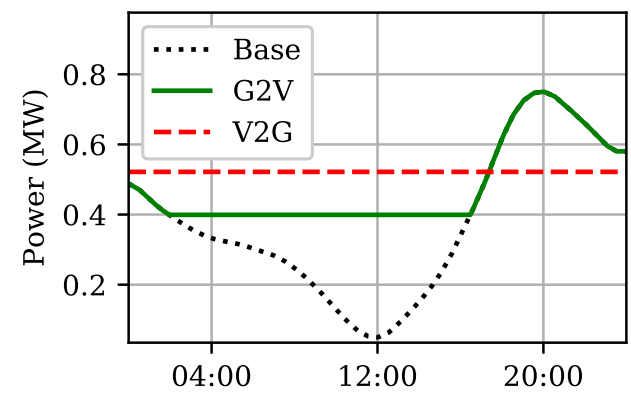

(b) Texas

Figure 7: An example of total feeder load under both charging schemes. The dotted line shows the demand before EV charging was added, the solid line shows the total load with optimal G2V charging, and the dotted line shows optimal V2G charging.

line shows the demand before EV charging was added, the solid line shows the total load with optimal G2V charging, and the dotted line shows optimal $\mathrm{V} 2 \mathrm{G}$ charging. In the UK case the optimal profiles are identical, because the EV load is large enough to completely flatten the profile without V2G. Whereas, in the Texas case the EV load was not sufficient to completely flatten load with G2V, so the peak demand can be reduced by using V2G to flatten total load.

In the Texas case, when $100 \%$ of households have an EV, a $15 \%$ reduction in peak demand is still possible. In part this result can be attributed to the industrial load on the network, which reduces the significance of the EV load relative to the existing demand on the network. The maximum increase in battery throughput coincides with the peak reduction in demand. However, it tapers away much faster, as the $\mathrm{V} 2 \mathrm{G}$ services are being split between a larger number of vehicles.

Figure 8 shows the change in losses for the same set of simulations. Again all simulations saw an increase in both charging and distribution losses, and the largest increase coincided with the greatest reduction in peak demand. The magnitude of the increase in total losses per household is similar to the UK case, however here the distribution losses made up a much more significant share. This makes sense, as the pre-existing load on the feeder is larger so the increase in load due to EV charging has a more significant effect. 


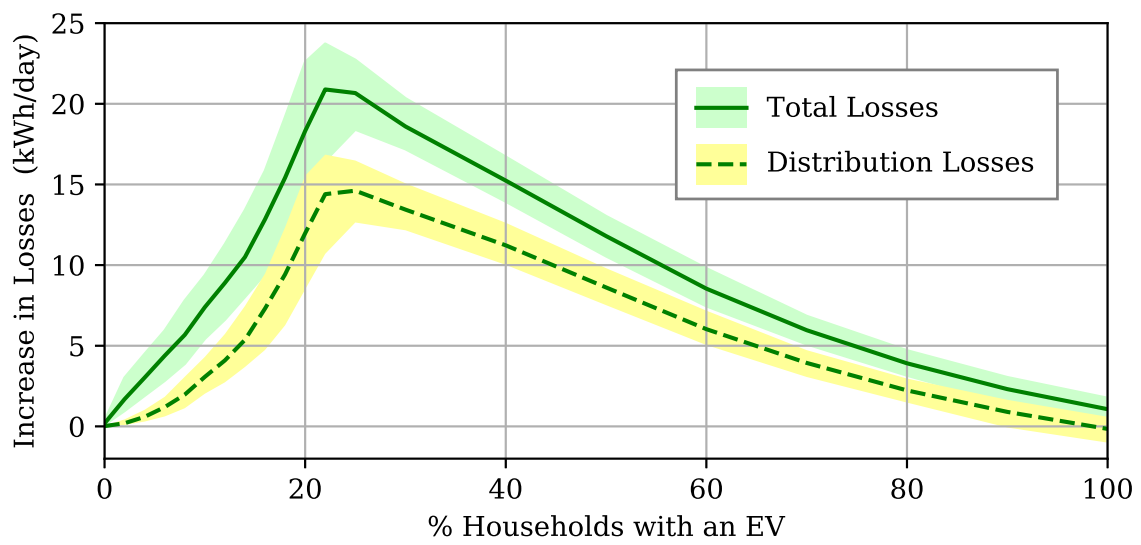

Figure 8: The increase in total losses and those just in the distribution system using bidirectional charging compared to uni-directional. The lines show the average values and the shaded areas cover the $90 \%$ confidence interval.

\section{Conclusions}

This paper quantified the costs and benefits of bi-directional smart charging of EVs in low voltage residential networks, compared to uni-directional smart charging. Two specific case studies were considered, one representing charging in a UK residential network and one representing a US network with a mix of residential and industrial load.

Bi-directional charging has the potential to reduce peak demand on a residential network compared to uni-direction charging; in the UK case study peak demand could be reduced by up to $35 \%$, and in the Texas case study by up to $20 \%$. However, the possible reduction depends heavily on the number of vehicles on the network. When there are too few vehicles, there is insufficient capacity to guarantee V2G at peak times, but each vehicle increases the total energy demand on the network. The greatest benefit was observed when between 10 and $20 \%$ of households have an EV. In the UK case, when $100 \%$ of households had an EV there was no additional benefit to bi-directional charging. Whereas in the Texas case (where there was a significant amount of solar generation) there was still additional benefit at $100 \% \mathrm{EV}$ penetration. This can be attributed to the larger difference between peak and trough in existing demand, caused by the combination higher household demand and solar generation.

However, the additional reduction in peak demand comes at a cost to the 
EV owner. Battery throughput was increased by up to $300 \%$, and this is likely to accelerate the degradation of the EVs' batteries. At smaller levels of EV penetration, when the $\mathrm{V} 2 \mathrm{G}$ services were being provided by a smaller number of vehicles, the increase is highest. As well as the degradation cost, the owner will also experience an increased electricity bill, due to losses in the charger. This cost is approximately linearly dependant on the amount of $\mathrm{V} 2 \mathrm{G}$ an EV provides.

The resistive losses in the distribution system are also increased by providing V2G services - the dominance of these losses compared to the charging losses depends on the network topology and existing load on the network. Resistive losses should be considered separately, as they are paid for by all customers on the network, regardless of whether they have an EV or not.

Finally, it should be noted that there were distinct differences in the two case studies considered. In the UK case there was only additional benefit to V2G at low penetration levels, and charging losses were more significant than distribution losses. Whereas, in the Texas case there was additional benefits at all levels of penetration, and the distribution losses dominated. This difference highlights the necessity of using representative load and network data in order to inform decisions about the viability of V2G in residential networks.

Two main areas of further work are identified. First, a higher fidelity model for battery degradation should be incorporated, so that a financial cost can be associated with the increase in battery throughput. Second, the case for V2G in emergency response services needs to be investigated - as it is likely that the benefits will be higher and the costs lower than in regular load flattening.

\section{Acknowledgements}

The authors would like to thank Jaguar Land Rover for their support in this research.

\section{References}

[1] Smart J, Schey S. Battery Electric Vehicle Driving and Charging Behavior Observed Early in The EV Project. SAE International Journal of Alternative Powertrains. 2012;1(1):27-33. 
[2] Hattam L, Greetham DV. Green neighbourhoods in low voltage networks: measuring impact of electric vehicles and photovoltaics on load profiles. J Mod Power Syst Clean Energy. 2017;5(1):105-116.

[3] Moses PS, Masoum MAS, Hajforoosh S. Overloading of distribution transformers in smart grid due to uncoordinated charging of plug-In electric vehicles. In: 2012 IEEE PES Innovative Smart Grid Technologies (ISGT); 2012. p. 1-6.

[4] Geth F, Leemput N, Roy JV, Buscher J, Ponnette R, Driesen J. Voltage droop charging of electric vehicles in a residential distribution feeder. In: 2012 3rd IEEE PES Innovative Smart Grid Technologies Europe (ISGT Europe); 2012. p. 1-8.

[5] UK Government Department for Business, Energy \& Industrial Strategy. Government funded electric car chargepoints to be smart by July 2019;. Accessed: 2019-04-16. https://www.gov.uk/government/news/ government-funded-electric-car-chargepoints-to-be-smart-by-july-2019.

[6] Benetti G, Delfanti M, Facchinetti T, Falabretti D, Merlo M. Real-Time Modeling and Control of Electric Vehicles Charging Processes. IEEE Transactions on Smart Grid. 2015;6(3):1375-1385.

[7] Gan L, Topcu U, Low SH. Optimal decentralized protocol for electric vehicle charging. IEEE Transactions on Power Systems. 2013;28(2):940951.

[8] Mets K, Verschueren T, De Turck F, Develder C. Exploiting V2G to optimize residential energy consumption with electrical vehicle (dis)charging. In: 2011 IEEE 1st International Workshop on Smart Grid Modeling and Simulation, SGMS 2011; 2011. .

[9] Fleet News. OVO installs its first domestic vehicle-to-grid charger; 2018. https://www.fleetnews.co.uk/news/fleet-industry-news/.

[10] Kumar Nunna HSVS, Battula S, Doolla S, Srinivasan D. Energy Management in Smart Distribution Systems with Vehicle-To-Grid Integrated Microgrids. IEEE Transactions on Smart Grid. 2018;9(5):4004-4016. 
[11] Moreira R, Ollagnier L, Papadaskalopoulos D, Strbac G. Optimal multiservice business models for electric vehicles. In: 2017 IEEE Manchester PowerTech; 2017. .

[12] Saber AY, Venayagamoorthy GK. Intelligent unit commitment with vehicle-to-grid: a cost-emission optimization. Journal of Power Sources. 2010;195(3):898 - 911. Available from: http://www.sciencedirect.com/ science/article/pii/S037877530901341X.

[13] Thingvad A, Ziras C, Marinelli M. Economic value of electric vehicle reserve provision in the Nordic countries under driving requirements and charger losses. Journal of Energy Storage. 2019 02;21:826-834.

[14] Kempton W, Tomić J. Vehicle-to-grid power fundamentals: Calculating capacity and net revenue. Journal of Power Sources. 2005;144(1):268279.

[15] Ma Y, Houghton T, Cruden A, Infield D. Modeling the benefits of vehicle-to-grid technology to a power system. IEEE Transactions on Power Systems. 2012;27(2):1012-1020.

[16] Andersson SL, Elofsson AK, Galus MD, Goransson L, Karlsson S, Andersson G. Plug-in hybrid electric vehicles as regulating power providers: Case studies of Sweden and Germany. Energy Policy. 2010;38(6):27512762 .

[17] Lopez MA, de la Torre S, Martin S, Aguado JA. Demand-side management in smart grid operation considering electric vehicles load shifting and vehicle-to-grid support. International Journal of Electrical Power \& Energy Systems. 2015;64:689 - 698. Available from: http: //www.sciencedirect.com/science/article/pii/S0142061514005006.

[18] Calearo L, Thingvad A, Ipsen H, Marinelli M. Economic Value and User Remuneration for EV Based Distribution Grid Services. In: Innovative Smart Grid Technologies (ISGT) Europe; 2019. .

[19] Zecchino A, Prostejovsky A, Ziras C, Marinelli M. Large-scale provision of frequency control via V2G: The Bornholm power system case. Electric Power Systems Research. 2019 05;170:25-34. 
[20] Yilmaz M, Krein PT. Review of the impact of vehicle-to-grid technologies on distribution systems and utility interfaces. IEEE Transactions on Power Electronics. 2013;28(12):5673-5689.

[21] Ortega-Vazquez MA. Optimal scheduling of electric vehicle charging and vehicle-to-grid services at household level including battery degradation and price uncertainty. IET Generation, Transmission \& Distribution. 2014;8(6):1007-1016.

[22] Uddin K, Jackson T, Widanage WD, Chouchelamane G, Jennings PA, Marco J. On the possibility of extending the lifetime of lithium-ion batteries through optimal V2G facilitated by an integrated vehicle and smart-grid system. Energy. 2017;133:710 - 722 .

[23] Thompson AW. Economic implications of lithium ion battery degradation for Vehicle-to-Grid (V2X) services. Journal of Power Sources. 2018;396:691 - 709. Available from: http://www.sciencedirect.com/ science/article/pii/S0378775318306499.

[24] Barre A, Deguilhem B, Grolleau S, Gerard M, Suard F, Riu D. A review on lithium-ion battery ageing mechanisms and estimations for automotive applications. Journal of Power Sources. 2013;241:680 - 689.

[25] Igualada L, Corchero C, Cruz-Zambrano M, Heredia FJ. Optimal energy management for a residential microgrid including a vehicle-to-grid system. IEEE Transactions on Smart Grid. 2014;5(4):2163-2172.

[26] Xu NZ, Chung CY. Reliability evaluation of distribution systems including vehicle-to-home and vehicle-to-grid. IEEE Transactions on Power Systems. 2016;31(1):759-768.

[27] Clement-Nyns K, Haesen E, Driesen J. The impact of vehicle-to-grid on the distribution grid. Electric Power Systems Research. 2011;.

[28] Feldman B, Tanner M, Rose C. Peak Demand Reduction Strategy. Navigant Consulting; 2015.

[29] Engineering IB. Energy Demand Research Project: Early Smart Meter Trials, 2007-2010.;. UK Data Service.

[30] Pecan Street inc. Spotlight; 2018. 
[31] Haustein S, Jensen AF. Factors of electric vehicle adoption: A comparison of conventional and electric car users based on an extended theory of planned behavior. International Journal of Sustainable Transportation. 2018;p. 1-13.

[32] Lepanjuuri K, Cornick P, Byron C, Templeton I, Hurn J. National Travel Survey: 2015 Report. Department for Transport; 2016.

[33] U S Department of Transportation. National Household Travel Survey 2009, 2015;.

[34] Data on Cars used for Testing Fuel Economy;. Accessed: 201705-15. https://www.epa.gov/compliance-and-fuel-economy-data/datacars-used-testing-fuel-economy.

[35] Electric Power Research Institute (EPRI). Hosting Capacity Feeders J1, K1, M1; 2019. Accessed Apr. 19. https://dpv.epri.com/feeder_models. html.

[36] Mohan N. Power Electronics: A First Course. Wiley; 2012.

[37] Thingvad A, Ziras C, Hu J, Marinelli M. Assessing the energy content of system frequency and electric vehicle charging efficiency for ancillary service provision. In: 2017 52nd International Universities Power Engineering Conference, UPEC 2017; 2017. p. 1-6.

[38] Deakin M, Crozier C, Morstyn T, Apostolopulou D, McCulloch M. Stochastic Hosting Capacity in Low Voltage Distribution Networks. In: IEEE PES General Meeting; 2019. https://arxiv.org/abs/1902.08780.

[39] Institite EPR. The Open Distirbution System Simulator (OpenDSS); 2018. http://smartgrid.epri.com/SimulationTool.aspx.

[40] Sears J, Roberts D, Glitman K. A comparison of electric vehicle Level 1 and Level 2 charging efficiency. SusTech. 2014;. 